\title{
BIALGEBRAIC CONTEXTS FROM DUALITIES
}

\author{
KEITH A. KEARNES and FRANK VOGT
}

(Received 6 December 1994; revised 15 June 1995)

Communicated by B. A. Davey

\begin{abstract}
In this paper we show that a bialgebraic context which arises from a duality in a fairly general way must arise from a duality between categories of modules. To show this, we give an elementary proof of Mitchell's Embedding Theorem for prevarieties.
\end{abstract}

1991 Mathematics subject classification (Amer. Math. Soc.): primary 08C05; secondary 18E20, 16A49.

\section{Introduction}

The analysis of data with non-numerical and non-statistical methods becomes more and more important in many applications. Contributing to this domain, the theory of Formal Concept Analysis elaborates a general understanding of data. In our paper, we investigate a connection of this understanding of data with a particular algebraic setting.

Formal Concept Analysis provides a set-theoretical model of concepts which mathematizes the philosophical understanding that a concept is a unit of thought consisting of two parts: the extension covering all objects belonging to the concept, and the intension consisting of all attributes abstracting from the concept (cf. $[6,11])$. The basic structure of the model is a formal context which is defined as a triple $(G, M, I)$, where $G$ and $M$ are sets and $I$ is a binary relation between $G$ and $M$. The elements of $G$ are interpreted as objects and those of $M$ as attributes. For every $g$ in $G$ and $m$ in $M$, the relation $(g, m) \in I$ is interpreted as the object $g$ has the attribute $m$. A formal concept of $(G, M, I)$ is a pair $(C, D)$ where $C$ is a subset of $G$ and $D$ is a subset of $M$ such that $D$ consists of the common attributes of the objects in $C$ and $C$ consists of all objects which have the attributes in $D$. Then $C$ is called the extent and $D$ is

The research of the first author was supported by a fellowship from the Alexander von Humboldt Stiftung (C) 1996 Australian Mathematical Society 0263-6115/96\$A2.00+0.00 
called the intent of the formal concept. The formal concepts of a formal context form a complete lattice if they are ordered by the subconcept-superconcept relation. The extents of a formal context form a closure system on the set $G$ of objects whereas the intents form a closure system on $M$.

After this short description of the conceptual language provided by Formal Concept Analysis, we describe how this language can be applied in a particular area of general algebra. In [10], it is described how this application can enrich the language of Formal Concept Analysis by algebraic constructions for purposes of data analysis. The basic idea is motivated by the formal context $\left(\boldsymbol{V}, V^{*}, \perp\right)$, where $V$ is a finite dimensional vector space, $\boldsymbol{V}^{*}$ is its dual space, and $v \perp \varphi$ is defined by $\varphi(v)=0$ for all $v$ in $V$ and all $\varphi$ in $V^{*}$. Then the following statement is a well known result in linear algebra:

The extents of the formal context $\left(\boldsymbol{V}, \boldsymbol{V}^{*}, \perp\right)$ are exactly the subspaces of $\boldsymbol{V}$ and the intents of $\left(\boldsymbol{V}, \boldsymbol{V}^{*}, \perp\right)$ are exactly the subspaces of $\boldsymbol{V}^{*}$.

A similar statement holds if we consider a finite abelian group $\boldsymbol{G}$, its dual $\boldsymbol{G}^{*}$, and define $g \perp \varphi$ by $\varphi(g)=1$. Recall that the dual of a (finite) abelian group $G$ is the group of all homomorphisms from $G$ into the unit circle of complex numbers, together with pointwise multiplication as group operation. In a general setting, we consider formal contexts $(\boldsymbol{A}, \boldsymbol{B}, \perp)$ where $\boldsymbol{A}$ and $\boldsymbol{B}$ are algebras in the sense of general algebra, and $\perp$ is an arbitrary relation between $A$ and $B$. There are two closure systems on $A$ : the closure system of extents of $(\boldsymbol{A}, \boldsymbol{B}, \perp)$ and the closure system of subuniverses of $\boldsymbol{A}$. In general, there is no connection between these closure systems, but it may happen that they coincide. A formal context $(\boldsymbol{A}, \boldsymbol{B}, \perp)$ is called a bialgebraic context if its extents are exactly the subuniverses of $\boldsymbol{A}$ and its intents are exactly the subuniverses of $\boldsymbol{B}$. In a bialgebraic context, the extents (intents) can be described in two different ways: conceptually, using the attributes in the set $B$ (objects in the set $A$ ), or algebraically, using the generation of subalgebras in the algebra $A(B)$. For more detailed information on bialgebraic contexts, see [9].

Next, we formulate what we understand by a duality for a prevariety of algebras. We follow the general approach introduced by D. M. Clark, B. A. Davey, H. Werner, and others (see, for example, $[2,3,4]$ ), but we restrict to the case where the structures in both categories are total algebras, with respect to the requirements of this paper. Let $\underline{P}:=(P, F)$ be an algebra of type $(\mathscr{F}, \sigma)$. By $\mathscr{P}:=\operatorname{ISP}(\underline{P})$ we denote the prevariety generated by $\underline{P}$. We consider $\mathscr{P}$ not only as a class but as a category of algebras, that is, as the full subcategory of the category of all algebras of type $(\mathscr{F}, \sigma)$. Now, we introduce an alter ego for the algebra $\underline{P}$. A topological structure $P:=(P, G, \tau)$ is called algebraic over $\underline{P}$ if the following conditions hold:

(i) $G$ is a family of operations on $P$ such that, if $g \in G$ is $n$-ary for $n \geq 0$, then $g: \underline{P^{n}} \longrightarrow \underline{P}$ is a homomorphism. Observe that this implies that $\{g\}$ is a subalgebra of $\underline{P}$ if $g$ is a constant. 
(ii) $\tau$ is a compact topology on $P$ such that if $f \in F$ is $n$-ary then $f: P^{n} \longrightarrow P$ is continuous with respect to the product topology on $P^{n}$.

Let $\mathscr{Q}:=I S_{c} \mathrm{P}(\underset{\sim}{P})$ be the class of all isomorphic (that is, simultaneously algebraically isomorphic and homeomorphic) copies of topologically closed substructures of powers of $\underset{\sim}{P}$. Again, we may consider $\mathscr{Q}$ as a category of topological structures with the continuous homomorphisms as morphisms. Now we are ready to establish a duality between between $\mathscr{P}$ and $\mathscr{Q}$.

For every algebra $A \in \mathscr{P}$, let $D(\boldsymbol{A}) \subseteq P^{A}$ be the set of all homomorphisms from $A$ into $\underline{P}$, endowed with the pointwise defined operations from $G$ and the subspace topology generated by $\tau$. For every homomorphism $\theta: \boldsymbol{A} \longrightarrow \boldsymbol{B}$ in $\mathscr{P}$ we define $D(\theta): D(\boldsymbol{B}) \longrightarrow D(\boldsymbol{A})$ by $(D(\theta))(\psi):=\psi \circ \theta$ for all $\psi \in D(\boldsymbol{B})$. Then $D(\boldsymbol{A})$ is in $\mathscr{Q}$ and $D(\theta)$ is a continuous homomorphism. Analogously, for all $\boldsymbol{X} \in \mathscr{Q}$, we define $E(X) \subseteq P^{X}$ as the set of all continuous homomorphisms from $X$ into $P$, endowed with the pointwise defined operations from $F$. For every continuous homomorphism $\omega: \boldsymbol{X} \longrightarrow \boldsymbol{Y}$ in $\mathscr{Q}$ let $E(\omega): E(\boldsymbol{Y}) \longrightarrow E(\boldsymbol{X})$ be given by $(E(\omega))(\mu):=\mu \circ \omega$ for all $\mu \in E(\boldsymbol{Y})$. Then $E(X)$ is in $\mathscr{P}$ and $E(\omega)$ is a homomorphism.

With these definitions, $D$ and $E$ are contravariant functors. It turns out that they form a dual adjunction where the units of the adjunction are given by the natural evaluation maps

$$
\begin{array}{ll}
\eta_{\boldsymbol{A}}: \boldsymbol{A} \longrightarrow E D(\boldsymbol{A}), & \left(\eta_{\boldsymbol{A}}(a)\right)(\varphi):=\varphi(a) \quad \text { and } \\
\varepsilon_{\boldsymbol{X}}: \boldsymbol{X} \longrightarrow D E(\boldsymbol{X}), & \left(\varepsilon_{\boldsymbol{X}}(x)\right)(\kappa):=\kappa(x) .
\end{array}
$$

for all $\boldsymbol{A} \in \mathscr{P}, a \in A, \varphi \in D(\boldsymbol{A}), \boldsymbol{X} \in \mathscr{Q}, x \in X$, and $\kappa \in E(\boldsymbol{X})$. It is immediate from the conditions concerning $\underline{P}$ and $\underset{\sim}{P}$ that $\eta_{A}$ and $\varepsilon_{X}$ are always injective and that $\varepsilon_{X}$ is a homeomorphism onto its image. Hence, $\eta_{A}$ and $\varepsilon_{X}$ are always embeddings. It depends on the structure of $\underline{P}$ and $\underset{\sim}{P}$ whether they are surjective, i. e., whether they are isomorphisms. We say that $\underset{\sim}{P}$ yields a duality on $\mathscr{P}$ if $\eta_{A}$ is an isomorphism for every $\boldsymbol{A} \in \mathscr{P}$. If, additionally, $\varepsilon_{\boldsymbol{X}}$ is an isomorphism for all $\boldsymbol{X} \in \mathscr{Q}$, the duality is called full.

Pontryagin duality between discrete and compact abelian groups and Morita duality between certain categories of left and right modules can be considered as dualities of the type described above. The construction of the bialgebraic contexts for finite dimensional vector spaces and for finite abelian groups which was mentioned earlier, is closely connected to these dualities. Therefore, we can ask whether there is a general method of construction of bialgebraic contexts using dualities. Before we can state the result we must introduce some additional notions.

Let $\mathscr{C}$ be a category of algebras (with or without topology) which has a zero object. Let $\boldsymbol{A} \in \mathscr{C}$ and let $\iota: \boldsymbol{C} \longrightarrow \boldsymbol{A}$ be a morphism in $\mathscr{C}$. Then $\iota$ is called a normal monomorphism if it is the kernel of a suitable morphism $\varphi: A \longrightarrow B$, that is, the 
equalizer of $\varphi$ and the unique zero morphism $0_{A B}$. An easy argument shows that such an equalizer must be a monomorphism. Therefore, the pair $(C, \iota)$ is a subobject of $\boldsymbol{A}$ which, since $\iota$ is normal, will be called a normal subobject. Dually, a morphism $\sigma: \boldsymbol{A} \longrightarrow \boldsymbol{C}$ is called a conormal epimorphism if it is the cokernel of a suitable morphism $\varphi: \boldsymbol{B} \longrightarrow \boldsymbol{A}$, that is, the coequalizer of $\varphi$ and $0_{\boldsymbol{B A}}$. The pair $(\sigma, \boldsymbol{C})$ is called a conormal quotient object. It is not hard to show that a monomorphism is normal if and only if it is the kernel of its cokernel and that an epimorphism is conormal if and only if it is the cokernel of its kernel. We will call a category normal if every monomorphism is normal and we will call it conormal if every epimorphism is conormal. These concepts require that the category has a zero object.

Now we interpret these concepts for a prevariety $\mathscr{P}$. As is common, we identify subobjects with subalgebras. A congruence is a compatible equivalence relation. If $\boldsymbol{A} \in \mathscr{P}$ and $\theta$ is the congruence associated with the kernel of a homomorphism of $A$ onto another member of $\mathscr{P}$, then we call $\theta$ a relative congruence of $A$. If $\mathscr{P}$ is a prevariety with a zero object, $Z$, then each $\boldsymbol{A} \in \mathscr{P}$ has an element 0 which is the image of $0_{Z A}$. (The element 0 does not depend on the choice of $Z$.) In this situation, the normal subobjects of $\boldsymbol{A}$ correspond to subalgebras of $\boldsymbol{A}$ which equal the 0 -class of some relative congruence of $\boldsymbol{A}$. A conormal epimorphism with domain $\boldsymbol{A}$ has kernel $\theta$ which is a relative congruence with the following property: $\theta$ is the least relative congruence on $\boldsymbol{A}$ which has $0 / \theta$ as a class.

Now we can state a theorem from [9] which generalizes the construction of bialgebraic contexts for vector spaces and abelian groups.

THEOREM 1.1. Let $P$ yield a duality on $\mathscr{P}$ such that there is a constant 0 in $F \cap G$ which is idempotent in $\underset{\sim}{P}$ ( that is, $g(0, \ldots, 0)=0$ for all $g \in G$ ). For every algebra $\boldsymbol{A}$ in $\mathscr{P}$, let $\mathbb{K}(\boldsymbol{A}):=(\boldsymbol{A}, D(\boldsymbol{A}), \perp)$, where, for all $a \in A$ and $\varphi \in D(\boldsymbol{A})$,

$$
a \perp \varphi \text { if and only if } \varphi(a)=0 .
$$

Then $\mathbb{K}(\boldsymbol{A})$ is a bialgebraic context if and only if all subalgebras of $\boldsymbol{A}$ and all subalgebras of $D(\boldsymbol{A})$ are normal.

In [9], an algebra $\boldsymbol{A}$ in a category $\mathscr{C}$ is called Hamiltonian relative to $\mathscr{C}$ if every subalgebra of $\boldsymbol{A}$ is normal.

In this paper we characterize all situations in the previous theorem where $P$ is finite and $\mathbb{K}(\boldsymbol{A})$ is a bialgebraic context for all finitely generated $\boldsymbol{A} \in \mathscr{P}$. The main result is that $\mathscr{P}$ must be term equivalent to a variety of modules.

\section{Varieties of modules}

Our purpose in this section is to show that a general method of constructing bialgebraic contexts from a duality must arise from a full duality between a variety 
of modules and a second category. For the main proof we do not need a deep understanding of duality theory as it is developed in [4]. All that we need to know is that, by a duality for the pair $\langle\mathscr{P}, \mathscr{Q}\rangle$ of a prevariety $\mathscr{P}$ and a category $\mathscr{Q}$ of algebras, we mean at least the following:

(i) There is a contravariant right adjunction $D: \mathscr{P} \longrightarrow \mathscr{Q}$ and $E: \mathscr{Q} \longrightarrow \mathscr{P}$.

(ii) The units of this adjunction are $\eta: I \longrightarrow E D$ and $\epsilon: I \longrightarrow D E$ and $\eta$ is a natural isomorphism while $\epsilon$ is a natural monomorphism.

Our assumptions will be that $\mathscr{P}$ and $\mathscr{Q}$ satisfy these two conditions and that $\mathscr{P}$ is normal and conormal. Let us explain why we make these normality assumptions.

By having a 'general method of constructing bialgebraic contexts from a duality' we mean that we have a pair $\langle\mathscr{P}, \mathscr{Q}\rangle$ for which there is a duality and that, for 'many' members $\boldsymbol{A} \in \mathscr{P}$, we have that $\mathbb{K}(\boldsymbol{A}):=(\boldsymbol{A}, D(\boldsymbol{A}), \perp)$ is a bialgebraic context. Since the notion of bialgebraic contexts arises from data analysis, one realistically only needs to consider finitely many finite members of $\mathscr{P}$, although we want the freedom to choose among many different finite members of $\mathscr{P}$ to select an appropriate one for a specific problem. Hence, it is natural to restrict attention to the case where $\mathscr{P}=\operatorname{ISP}(\underline{P})$ with $\underline{P}$ finite and for each finite $A \in \mathscr{P}$ it is the case that $\mathbb{K}(\boldsymbol{A})$ is bialgebraic.

LEMMA 2.1. Under the assumptions of Theorem 1.1 , let $\mathscr{P}$ be generated by a finite algebra $\underline{P}$, and let $\mathbb{K}(\boldsymbol{A})$ be a bialgebraic context for every finite $\boldsymbol{A} \in \mathscr{P}$. Then $\mathscr{P}$ is a normal and conormal quasivariety.

PROOF. By Theorem 1.1, we get that all subalgebras of finite members of $\mathscr{P}$ are normal. Since $\underline{P}$ is finite, $\mathscr{P}=\operatorname{ISP}(\underline{P})$ is a locally finite quasivariety. In particular, $\mathscr{P}$ is closed under ultraproducts. Now, to show that $\mathscr{P}$ is normal we must show that if $\boldsymbol{A} \in \mathscr{P}$ and $\boldsymbol{S}$ is a subalgebra of $\boldsymbol{A}$, then there is a relative congruence $\theta$ such that $S=0 / \theta$. For $X \subseteq A$, let $\boldsymbol{B}_{X}$ denote the subalgebra generated by $X$. Since $\mathscr{P}_{\text {fin }}$ is normal, for each finite $X \subseteq A$ we have that $S \cap B_{X}$ is the 0-class of a relative congruence of $\boldsymbol{B}_{X}$. Hence there is a homomorphism $\phi_{X}$ of $\boldsymbol{B}_{X}$ onto a member of $\mathscr{P}$ where $\phi_{X}\left(S \cap B_{X}\right)=\{0\}$ while $\phi_{X}(b) \neq 0$ if $b \in B_{X}-S$. If $I$ denotes the set of nonempty finite subsets of $A$, then

$$
\mathscr{F}:=\left\{Z_{X} \mid X \in I, Z_{X}:=\{Y \in I \mid Y \supseteq X\}\right\}
$$

is a filter on $I$. Let $\mathscr{U}$ be an ultrafilter extending $\mathscr{F}$. There is an embedding $\mu$ of $\boldsymbol{A}$ into the ultraproduct $\left(\prod_{X \in I} \boldsymbol{B}_{X}\right) / \mathscr{U}$ described by $\mu(x)=\bar{u} / \mathscr{U}$ where $u_{X}=x$ if $x \in B_{X}$ and $u_{X}=0$ if $x \notin B_{X}$. The composite homomorphism $\left(\left(\prod_{X \in I} \phi_{X}\right) / \mathscr{U}\right) \circ \mu$ is a homomorphism from $A$ to a member of $\mathscr{P}$ which is zero on $S$ but not zero on any $a \in A-S$. Hence the kernel of this homomorphism is a relative congruence whose 0 -class is exactly $S$. This proves the normality of $\mathscr{P}$. 
In order to prove that $\mathscr{P}$ is conormal, we show first that the subcategory $\mathscr{Q}_{\text {fin }}$ of finite members of $\mathscr{Q}$ is normal. Any finite $\boldsymbol{B} \in \mathscr{Q}$ has an embedding $\epsilon_{\boldsymbol{B}}: \boldsymbol{B} \longrightarrow D E(\boldsymbol{B})$. The algebra $E(\boldsymbol{B}) \in \mathscr{P}$ is finite, hence $\mathbb{K}(E(\boldsymbol{B}))$ is bialgebraic by assumption. Theorem 1.1 implies that the subalgebras of $D E(B)$ are normal. Since normality of all subalgebras is inherited by subalgebras and $B$ is isomorphic to a subalgebra of $D E(\boldsymbol{B})$, the subalgebras of $\boldsymbol{B}$ are normal. This holds for all finite $\boldsymbol{B} \in \mathscr{Q}$, hence $\mathscr{Q}_{\text {fin }}$ is normal. The next step is to prove that $\mathscr{P}_{\text {fin }}$ is conormal. Since the duality for $\langle\mathscr{P}, \mathscr{Q}\rangle$ restricts to a duality for $\left\langle\mathscr{P}_{\text {fin }}, \mathscr{Q}_{\text {fin }}\right\rangle$, it suffices to show that a duality for $\left\langle\mathscr{P}_{\text {fin }}, \mathscr{Q}_{\text {fin }}\right\rangle$ where both categories are normal implies that $\mathscr{P}_{\text {fin }}$ is conormal. This is proved below in Lemma 2.2. Assuming this, it is easy to see that $\mathscr{P}$ itself is conormal. For if not then some $C \in \mathscr{P}$ has a relative congruence $\beta$ which is not the least relative congruence whose 0 -class is $0 / \beta$. If $\alpha$ is another relative congruence with $0 / \alpha=0 / \beta$ and $\beta \not \alpha$, then there is a pair of elements $(p, q) \in \beta-\alpha$. Let $D$ be the subalgebra of $C$ generated by $\{p, q\}$. Then since $\left.(p, q) \in \beta\right|_{D}-\left.\alpha\right|_{D}$ the congruences $\left.\alpha\right|_{D}$ and $\left.\beta\right|_{D}$ are relative congruences of $\boldsymbol{D}$ whose 0 -classes are both equal to $D \cap(0 / \alpha)=D \cap(0 / \beta)$. Since $\left.(p, q) \in \beta\right|_{D}-\left.\alpha\right|_{D}$ we get that $\left.\beta\right|_{D}$ is a relative congruence of $\boldsymbol{D}$ which is not the least relative congruence whose 0 -class is $0 /\left.\beta\right|_{D}$. This is impossible if $\mathscr{P}_{\text {fin }}$ is conormal since $D \in \mathscr{P}_{\text {fin }}$. We conclude that $\mathscr{P}$ is conormal if $\mathscr{P}_{\text {fin }}$ is conormal.

To prove that $\mathscr{P}_{\text {fin }}$ is conormal we must show that every epimorphism in $\mathscr{P}_{\text {fin }}$ is the cokernel of its kernel. A minimal requirement for this is that every epimorphism has a kernel. This holds for categories of the form $\mathscr{P}_{\text {fin }}$ when $\mathscr{P}$ is a prevariety with zero, since such categories have equalizers. It turns out that this is the only special property of prevarieties that is needed to prove that $\mathscr{P}_{\text {fin }}$ is conormal. Therefore, we formulate the following lemma in a more general way.

LeMMA 2.2. Assume that $D: \mathscr{P} \longrightarrow \mathscr{Q}$ and $E: \mathscr{Q} \longrightarrow \mathscr{P}$ is a contravariant right adjunction between normal categories $\mathscr{P}$ and $\mathscr{Q}$ where the unit $\eta: I \longrightarrow E D$ is a natural isomorphism and the unit $\epsilon: I \longrightarrow D E$ is a natural monomorphism. If epimorphisms in $\mathscr{P}$ have kernels, then $\mathscr{P}$ is conormal.

PROOF. The statement of this lemma would be entirely obvious if we knew $\epsilon$ was a natural isomorphism, since conormality is the categorical dual of normality. However, our assumptions do not mean that $\mathscr{P}$ and $\mathscr{Q}$ are dual categories.

It suffices to prove the lemma in the case when $\mathscr{P}$ and $\mathscr{Q}$ are skeletal categories (that is, when the only isomorphisms are identities), and with this assumption we get that each component of $\eta$ is an identity. This simplifies notation a bit. For example, if $\varphi: \boldsymbol{U} \longrightarrow \boldsymbol{V}$ in $\mathscr{P}$, then we can immediately replace the double dual $E D(\varphi): E D(\boldsymbol{U}) \longrightarrow E D(\boldsymbol{V})$ with $\varphi: \boldsymbol{U} \longrightarrow \boldsymbol{V}$.

Since $D: \mathscr{P} \longrightarrow \mathscr{Q}$ and $E: \mathscr{Q} \longrightarrow \mathscr{P}$ is a contravariant right adjunction, both $D$ and $E$ convert colimits into limits. Since zero objects are colimits and limits, both 
$D$ and $E$ preserve zero objects. It follows that both $D$ and $E$ preserve zero morphisms (reversing direction), since these are exactly the morphisms that factor through zero objects. Both $D$ and $E$ convert coequalizers into equalizers, so they convert cokernels into kernels. The fact that both $\eta$ and $\epsilon$ are natural monomorphisms implies that both $D$ and $E$ are faithful functors. (If, for example, $\alpha, \beta: \boldsymbol{A} \longrightarrow \boldsymbol{B}$ and $E(\alpha)=E(\beta)$, then

$$
\epsilon_{B} \circ \alpha=D E(\alpha) \circ \epsilon_{A}=D E(\beta) \circ \epsilon_{A}=\epsilon_{B} \circ \beta .
$$

Cancelling the monomorphism $\epsilon_{B}$ on the left yields $\alpha=\beta$.) Using the faithfulness of $E$ and the fact that $\eta$ is a natural isomorphism, it is easy to prove that $D$ converts epimorphisms into monomorphisms and monomorphisms into epimorphisms.

We must show that every epimorphism in $\mathscr{P}$ is the cokernel of its kernel. Let $\sigma: \boldsymbol{A} \longrightarrow \boldsymbol{B}$ be an epimorphism and let $\kappa: \boldsymbol{K} \longrightarrow \boldsymbol{A}$ be the kernel of $\sigma$ (which we have assumed to exist). If $\gamma: \boldsymbol{A} \longrightarrow \boldsymbol{C}$ is the cokernel of $\kappa$, then there is a natural morphism $\gamma^{\prime}: \boldsymbol{C} \longrightarrow \boldsymbol{B}$ such that $\gamma^{\prime} \circ \gamma=\sigma$. ( $\gamma^{\prime}$ exists since $\gamma$ is the cokernel of $\kappa$ and $\sigma \circ \kappa=0_{K B}$. The latter holds because $\kappa$ is the kernel of $\sigma$.) This gives us the following diagram

$$
\boldsymbol{K} \stackrel{\kappa}{\longrightarrow} \boldsymbol{A} \stackrel{\gamma}{\longrightarrow} \boldsymbol{C} \stackrel{\gamma^{\prime}}{\longrightarrow} \boldsymbol{B} .
$$

Our goal is to prove that $\gamma^{\prime}$ is an isomorphism (and therefore, since our categories are skeletal, that $\gamma^{\prime}$ is an identity). This will prove that $\sigma\left(=\gamma^{\prime} \circ \gamma=\gamma\right)$ is the cokernel of the kernel of $\sigma$.

Applying the functor $D$ to the previous situation, we get

$$
D(\boldsymbol{B}) \stackrel{D\left(\gamma^{\prime}\right)}{\longrightarrow} D(\boldsymbol{C}) \stackrel{D(\gamma)}{\longrightarrow} D(\boldsymbol{A}) \stackrel{D(x)}{\longrightarrow} D(\boldsymbol{K}) .
$$

$D(\sigma)=D(\gamma) \circ D\left(\gamma^{\prime}\right)$ is a monomorphism, since $D$ converts epimorphisms into monomorphisms. Since $\mathscr{Q}$ is normal, there exists a cokernel $\delta: D(\boldsymbol{A}) \longrightarrow \boldsymbol{F}$ of $D(\sigma)$. Since $E$ converts cokernels into kernels, $E(\delta): E(\boldsymbol{F}) \longrightarrow E D(\boldsymbol{A})=\boldsymbol{A}$ is the kernel of $\sigma: \boldsymbol{A} \longrightarrow \boldsymbol{B}$. Kernels are unique in skeletal categories, so $E(\boldsymbol{F})=\boldsymbol{K}$ and $E(\delta)=\kappa$. Our diagram now looks like

$$
D(\boldsymbol{B}) \stackrel{D\left(\gamma^{\prime}\right)}{\longrightarrow} D(\boldsymbol{C}) \stackrel{D(\gamma)}{\longrightarrow} D(\boldsymbol{A}) \stackrel{\delta}{\longrightarrow} \boldsymbol{F} \stackrel{\delta^{\prime}}{\longrightarrow} D E(\boldsymbol{F})=D(\boldsymbol{K}) .
$$

Taking the double dual of $\delta: D(\boldsymbol{A}) \longrightarrow \boldsymbol{F}$ we get the following commutative diagram.

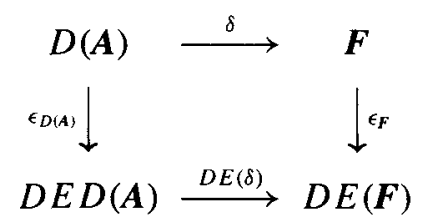


Since our categories are skeletal, $E D(\boldsymbol{A})=\boldsymbol{A}$ (so $D E D(\boldsymbol{A})=D(\boldsymbol{A})$ ). As observed earlier, $E(\delta)=\kappa$ (so $D E(\delta)=D(\kappa)$ ), and $E(\boldsymbol{F})=\boldsymbol{K}$ (so $D E(\boldsymbol{F})=D(\boldsymbol{K})$ ). Furthermore, $\epsilon_{D(A)}=\mathrm{id}_{D(A)}$. The previous diagram is just

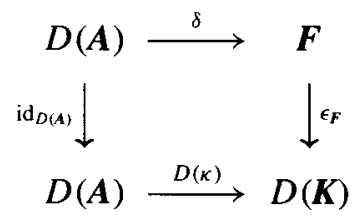

But now $\epsilon_{F}$ satisfies the definition of $\delta^{\prime}$, so $\delta^{\prime}=\epsilon_{F}$. This proves that $\delta^{\prime}$ is a monomorphism.

Since $\mathscr{Q}$ is normal and $D(\sigma): D(\boldsymbol{B}) \longrightarrow D(\boldsymbol{A})$ is a monomorphism with cokernel $\delta$, we get that $D(\sigma)$ is the kernel of $\delta . D(\gamma)$ has the property that

$$
0_{D(C) D(K)}=D\left(0_{K C}\right)=D(\gamma \circ \kappa)=D(\kappa) \circ D(\gamma)=\delta^{\prime} \circ \delta \circ D(\gamma) .
$$

Since $\delta^{\prime} \circ \delta \circ D(\gamma)=0_{D(C) D(K)}=\delta^{\prime} \circ 0_{D(C) F}$ and $\delta^{\prime}$ is a monomorphism, we can cancel $\delta^{\prime}$ from the left and get that $\delta \circ D(\gamma)=0_{D(C) F}$. This proves that $D(\gamma)$ is contained in the kernel of $\delta$, which is $D(\sigma)$, so there is a unique homomorphism $\lambda: D(\boldsymbol{C}) \longrightarrow D(\boldsymbol{B})$ with the property that $D(\gamma)=D(\sigma) \circ \lambda$. To summarize, we have shown that

- $D(\sigma)=D(\gamma) \circ D\left(\gamma^{\prime}\right)$.

- $D(\gamma)=D(\sigma) \circ \lambda$.

- $D(\sigma)$ is a monomorphism.

The first two items imply that $D(\sigma)=D(\sigma) \circ \lambda \circ D\left(\gamma^{\prime}\right)$. The third item allows us to cancel $D(\sigma)$ on the left to obtain that $\operatorname{id}_{D(B)}=\lambda \circ D\left(\gamma^{\prime}\right)$. Now since $D$ converts cokernels into kernels, $D(\gamma)$ is the kernel of $D(\kappa)$. Hence we also have

- $D(\gamma)$ is a monomorphism.

A similar argument using this item and the first two from above yields that $\mathrm{id}_{D(C)}=$ $D\left(\gamma^{\prime}\right) \circ \lambda$. Hence, $\lambda$ and $D\left(\gamma^{\prime}\right)$ are inverse isomorphisms. Since $\mathscr{Q}$ is skeletal, we have that $D(\boldsymbol{B})=D(\boldsymbol{C})$ and $D\left(\gamma^{\prime}\right)=\lambda=\mathrm{id}_{D(B)}$. Hence, $\gamma^{\prime}=E D\left(\gamma^{\prime}\right)=E\left(\mathrm{id}_{D(\boldsymbol{B})}\right)=$ $\mathrm{id}_{\boldsymbol{B}}$. This proves that $\sigma$ is the cokernel of its kernel.

We learn from Lemma 2.1 and Lemma 2.2 , that it is reasonable to assume that $\mathscr{P}$ is a normal and conormal prevariety if we consider dualities in connection with bialgebraic contexts. Although the discussion so far involved locally finite prevarieties, we do not use this assumption in the remainder of this section. 
LEMMA 2.3. A normal, conormal prevariety is an abelian category.

PROOF. There are varying sets of axioms which define abelian categories. One set, due to P. Freyd, is the following: $\mathscr{P}$ is abelian if and only if it is normal, conormal and has pullbacks and pushouts. (See Theorem I.20.1 of [8].) Clearly, any prevariety has pullbacks and pushouts, so we are done.

THEOREM 2.4. Let $\mathscr{P}$ be a category of algebras. The following conditions are equivalent.

(1) $\mathscr{P}$ is a prevariety and an abelian category.

(2) $\mathscr{P}$ is term equivalent to a variety of modules.

PROOF. Of course, any variety of modules is a prevariety and an abelian category. Conversely, assume that $\mathscr{P}$ is a prevariety and an abelian category. We now refer to Theorem IV.4.1 of [8] which contains a version of Mitchell's Embedding Theorem for abelian categories. We only state the part of this theorem that concerns us:

THEOREM 2.5. The following are equivalent for a category $\mathscr{P}$ :

(1) $\mathscr{P}$ is a cocomplete abelian category with a small projective generator.

(2) $\mathscr{P}$ is categorically equivalent to a variety of modules.

If one checks the definitions of cocomplete [8, p. 46], generator [8, pp. 71-72], and small object $[8$, p. 74$]$, then one finds that any prevariety $\mathscr{P}$ is a cocomplete category for which the free algebra $\boldsymbol{F}_{\mathscr{g}}(1)$ is a small generator. Free algebras in a category of algebras are projective if and only if epimorphisms are surjective, but in any abelian category of algebras this is the case. Hence, any prevariety which is an abelian category is categorically equivalent to a variety of modules.

There are many ways of proving that a prevariety which is categorically equivalent to a a variety of modules is term equivalent to a variety of modules. For example, it is not hard to show that a prevariety which is categorically equivalent to a variety must itself be a variety since a variety is nothing more than an exact prevariety and exactness is a categorical property. Furthermore, a variety which is categorically equivalent to a variety of modules is congruence modular, abelian and has a zero object. Now Theorem 9.16 of [5] applies to show that such a variety is term equivalent to a variety of modules.

The proof that $\mathscr{P}$ is term equivalent to a variety of modules that we have given is quite simple, except that it requires Mitchell's Embedding Theorem, which is deep. We now proceed to give an entirely elementary proof that $\mathscr{P}$ is term equivalent to a variety of modules. This second proof requires no knowledge of the structure 
of abelian categories. In particular, what follows might be viewed as saying that Mitchell's Theorem is elementary for prevarieties.

The collection of relative congruences on $\boldsymbol{B} \in \mathscr{P}$ is closed under complete intersection, so it makes sense to refer to the relative congruence generated by a set of pairs; it is the intersection of all relative congruences containing those pairs. The proof of the following lemma is obvious from the definitions of normality and conormality.

LEMMA 2.6. (See [8, Proposition I.14.2].) If $\mathscr{P}$ is a normal, conormal prevariety and $\boldsymbol{A} \in \mathscr{P}$, then the following mappings describe inverse bijections between the subuniverses of $\boldsymbol{A}$ and the relative congruences on $\boldsymbol{A}$.

(i) To each subuniverse $S$ assign the relative congruence $\theta$ generated by $S \times S$.

(ii) To each relative congruence $\theta$ assign $S=0 / \theta$.

For each $\boldsymbol{A} \in \mathscr{P}$, let $\Delta$ (or $\Delta_{\boldsymbol{A}}$ ) denote the relative congruence on $\boldsymbol{A}^{2}$ generated by $D \times D$ where $D=\{(x, x) \mid x \in A\}$ is the diagonal of $A^{2}$. One observes that the relative congruence $\Delta$ is a complement to each of the coordinate projection kernels of $\boldsymbol{A}^{2}$. For if $\pi_{0}$ and $\pi_{1}$ denote the coordinate projection kernels, then we have

$$
1_{A^{2}}=\pi_{i} \circ \Delta \circ \pi_{i}=\pi_{i} \vee \Delta .
$$

On the other hand, the subuniverses of $A^{2}$ corresponding to $\pi_{0}, \pi_{1}$ and $D$ which are guaranteed by Lemma 2.6 are $\{0\} \times A, A \times\{0\}$ and $D$ respectively. Since $D \cap(\{0\} \times A)=\{(0,0)\}$ and $\{(0,0)\}$ corresponds to the relative congruence $0_{A^{2}}$, we get that $\Delta \wedge \pi_{0}=0_{A^{2}}$. Similarly, $\Delta \wedge \pi_{1}=0_{A^{2}}$.

For a homomorphism $\varphi: A \longrightarrow B$ we define $\varphi_{\Delta}: A^{2} / \Delta \longrightarrow B^{2} / \Delta$ by $\varphi_{\Delta}((a, b) / \Delta)=(\varphi(a), \varphi(b)) / \Delta$.

\section{LEMMA 2.7. The construction $\boldsymbol{A} \mapsto \boldsymbol{A}^{2} / \Delta, \varphi \mapsto \varphi_{\Delta}$ is functorial.}

PROOF. We defined $\varphi_{\Delta}$ precisely so that it makes the required diagram commute. The only thing that needs to be checked is that, if $\varphi: A \longrightarrow \boldsymbol{B}$, then $\varphi_{\Delta}$ is well defined. This requires proving that the following implication holds:

$$
(a, b) \Delta_{A}(c, d) \text { implies }(\varphi(a), \varphi(b)) \Delta_{B}(\varphi(c), \varphi(d)) .
$$

In other words, we must show that $\Delta_{A} \subseteq(\varphi \times \varphi)^{-1}\left(\Delta_{B}\right)$. This follows since $(\varphi \times$ $\varphi)^{-1}\left(\Delta_{B}\right)$ is obviously a relative congruence of $\boldsymbol{A}^{2}$ which contains the generators of $\Delta_{A}$.

We denote by $\Delta$ the functor of Lemma 2.7. We now describe two natural transformations to the functor $\Delta$. Let $I$ and $I^{2}$ denote the identity functor and the squaring functor, respectively. (The squaring functor is the functor $\boldsymbol{A} \mapsto \boldsymbol{A}^{2}, \varphi \mapsto \varphi^{2}$.) 
Let $v: I^{2} \longrightarrow \Delta$ be defined by choosing the $A$-component of $v$ to be the natural homomorphism

$$
v_{A}: A^{2} \longrightarrow A^{2} / \Delta:(a, b) \mapsto(a, b) / \Delta .
$$

Let $\iota: I \longrightarrow \Delta$ be defined by choosing the $A$-component of $\iota$ to be

$$
\iota_{\boldsymbol{A}}: \boldsymbol{A} \longrightarrow \boldsymbol{A}^{2} / \Delta: a \mapsto(a, 0) / \Delta .
$$

LEMMA 2.8. $v$ is a natural transformation; $\iota$ is a natural isomorphism.

PROOF. To prove that $v$ is a natural transformation we must show that for each morphism $\varphi: A \longrightarrow \boldsymbol{B}$ in $\mathscr{P}$ the following diagram commutes:

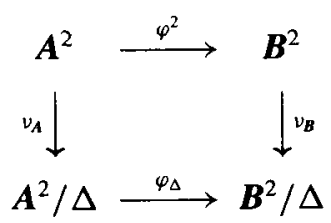

This is obvious from the definition of $\varphi_{\Delta}$.

To show that $\iota$ is a natural transformation we must show that

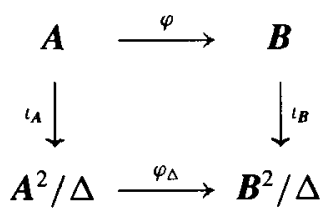

commutes. This requires proving that for every $a \in A$ we have $(\varphi(a), \varphi(0)) / \Delta=$ $(\varphi(a), 0) / \Delta$. But $\varphi(0)=0$, since in any member of $\mathscr{P}$ the element 0 interprets as the unique element which is in the image of a homomorphism from a zero object of $\mathscr{P}$.

To finish the proof of this lemma we must show that for any $\boldsymbol{A} \in \mathscr{P}$ the homomorphism $\iota_{\boldsymbol{A}}: \boldsymbol{A} \longrightarrow \boldsymbol{A}^{2} / \Delta$ is an isomorphism. The homomorphism $\iota_{\boldsymbol{A}}$ may be factored as $\alpha \circ \beta$ where $\beta: A \longrightarrow A^{2}: a \mapsto(a, 0)$ and $\alpha: A^{2} \longrightarrow A^{2} / \Delta$ is the natural homomorphism. The image of $\beta$ is a single $\pi_{1}$-class of $A^{2}$ and $\Delta$ is the congruence which is the kernel of $\alpha$. As we have observed, $\Delta \wedge \pi_{1}=0_{A^{2}}$, so $\Delta$ restricts trivially to $\beta(A)$ which means that the composite $\alpha \circ \beta$ is one-to-one.

Let $\boldsymbol{R}$ be the subalgebra of $\boldsymbol{A}^{2}$ whose universe consists of all pairs $\Delta$-related to some pair of $\beta(A)=A \times\{0\} . R$ contains $\{(0,0)\}$, so $R$ contains

$$
D=(0,0) / \Delta=\left\{(x, x) \in A^{2} \mid x \in A\right\} .
$$

Of course, $R$ also contains $A \times\{0\}$. Let $\rho$ be the relative congruence of $A^{2}$ which Lemma 2.6 associates with $R$. Since $D \subseteq R$ and $\Delta$ is the relative congruence 
associated with $D$, we get $\Delta \leq \rho$. Since $\pi_{1}$ is the relative congruence associated with $A \times\{0\}$, we get $\pi_{1} \leq \rho$. Therefore $1_{A^{2}}=\Delta \vee \pi_{1} \leq \rho$. This proves that $\rho=1_{A^{2}}$, so the subuniverse associated with $\rho$ (which is $R$ ) is all of $A^{2}$. From this we learn that every element of $A^{2}$ is $\Delta$-related to some element of $A \times\{0\}=\beta(A)$. If follows that the composite homomorphism $\iota_{A}: A \stackrel{\beta}{\longrightarrow} A^{2} \stackrel{\alpha}{\longrightarrow} A^{2} / \Delta$ is surjective. Thus, $\iota_{A}$ is an isomorphism, as claimed.

For a prevariety $\mathscr{P}$ we will call any natural transformation from the $n$-th power functor to the identity a natural $n$-ary operation. Lemma 2.8 proves that $\iota^{-1} \circ v$ : $I^{2} \longrightarrow I$ is a natural binary operation of $\mathscr{P}$. For a fixed choice of a zero object $Z \in \mathscr{P}$ we may define a functor $I^{0}$ by $\boldsymbol{A} \mapsto Z$ and $\varphi \mapsto \mathrm{id}_{Z}$. One natural zero-ary operation of $\mathscr{P}$ is the natural transformation $0: I^{0} \longrightarrow I$ whose $A$-component is the unique map from $\boldsymbol{Z}$ to $\boldsymbol{A}$. In symbols, $0_{A}=0_{\mathrm{ZA}}$. We now describe all of the natural operations of $\mathscr{P}$.

LEMMA 2.9. Let $\mathscr{P}$ be a prevariety and let $\sigma$ be a natural $n$-ary operation of $\mathscr{P}$. Then $\mathscr{P}$ has an $n$-ary term s which commutes with all terms of $\mathscr{P}$ and for each $\boldsymbol{A} \in \mathscr{P}$ we have

$$
\sigma_{\boldsymbol{A}}=s^{A}: \boldsymbol{A}^{n} \longrightarrow \boldsymbol{A}
$$

PROOF. Let $\boldsymbol{F}=\boldsymbol{F}_{\mathscr{P}}\left(x_{1}, \ldots, x_{n}\right)$ be the algebra in $\mathscr{P}$ which is freely generated by the set $\left\{x_{1}, \ldots, x_{n}\right\}$. The function $\sigma_{\boldsymbol{F}}: \boldsymbol{F}^{n} \longrightarrow \boldsymbol{F}$ is a homomorphism; so let $s$ be a term representing the element $\sigma_{F}\left(\left(x_{1}, \ldots, x_{n}\right)\right) \in F$. That is, choose $s$ so that $s^{F}\left(x_{1}, \ldots, x_{n}\right)=\sigma_{F}\left(\left(x_{1}, \ldots, x_{n}\right)\right)$. We claim that $s$ is the desired term.

Choose any $\boldsymbol{A} \in \mathscr{P}$, fix any $\left(a_{1}, \ldots, a_{n}\right) \in A^{n}$ and consider the homomorphism $\varphi: \boldsymbol{F} \longrightarrow \boldsymbol{A}$ determined by $x_{i} \mapsto a_{i}$. Since we have a commutative diagram

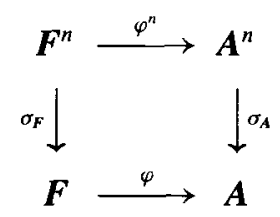

we can chase the element $\left(x_{1}, \ldots, x_{n}\right) \in F^{n}$ around both paths and obtain that

$$
\sigma_{A}\left(a_{1}, \ldots, a_{n}\right)=\varphi\left(s^{F}\left(x_{1}, \ldots, x_{n}\right)\right)=s^{A}\left(\varphi\left(x_{1}\right), \ldots, \varphi\left(x_{n}\right)\right)=s^{A}\left(a_{1}, \ldots, a_{n}\right) .
$$

Hence the values of $\sigma_{A}$ are identical with the values of $s^{A}$ for all $A \in \mathscr{P}$.

The term $s$ commutes with all other terms of $\mathscr{P}$, since for each $\boldsymbol{A} \in \mathscr{P}$ we have that $s^{A}=\sigma_{\boldsymbol{A}}: \boldsymbol{A}^{n} \longrightarrow \boldsymbol{A}$ is a homomorphism. 
Lemma 2.9 proves that every natural operation corresponds to a term of the same arity which commutes with all terms. Although we will not need the converse, it is true: any term $s$ which commutes with all terms defines a natural operation $\sigma$ by setting $\sigma_{A}=s^{A}$.

LEMMA 2.10. Let $\mathscr{P}$ be a prevariety which is normal and conormal. There are terms $O$ and $s$ which commute with all basic operations of $\mathscr{P}$ and satisfy the following equations:

(1) $s(x, x)=0$.

(2) $s(x, 0)=x$.

(3) $s(s(x, y), s(z, w))=s(s(x, z), s(y, w))$.

PROOF. We choose terms 0 and $s$ to be the terms guaranteed by Lemma 2.9 which correspond to the natural operations 0 and $\iota^{-1} \circ \nu$. For any $\boldsymbol{A} \in \mathscr{P}$ we have

$$
s^{A}(x, x)=\iota_{A}^{-1}\left(\nu_{A}(x, x)\right)=\iota_{A}^{-1}((x, x) / \Delta)=\iota_{A}^{-1}((0,0) / \Delta)=0 .
$$

Therefore (1) holds. Furthermore,

$$
s^{A}(x, 0)=\iota_{A}^{-1}\left(v_{A}(x, 0)\right)=\iota_{A}^{-1}((x, 0) / \Delta)=x
$$

so (2) holds. Finally, since $s$ is a term, but also a homomorphism from $\boldsymbol{A}^{2}$ to $\boldsymbol{A}$, we get that (3) holds.

THEOREM 2.11. Let $\mathscr{P}$ be a normal, conormal prevariety. Then $\mathscr{P}$ is term equivalent to a variety of modules.

ProOF. Define $x+y=s(x, s(0, y))$ and $-x=s(0, x)$. These terms commute with all terms of $\mathscr{P}$ since both 0 and $s$ have that property. We claim that $x+y,-x$ and 0 interpret as abelian group operations on any member of $\mathscr{P}$.

Clearly we have $x+0=s(x, s(0,0))=s(x, 0)=x$. Furthermore,

$$
\begin{aligned}
0+x & =s(0, s(0, x))=s(s(x, x), s(0, x)) \\
& =s(s(x, 0), s(x, x))=s(x, 0)=x .
\end{aligned}
$$

This proves the unit laws. We have $(-x)+x=s(s(0, x), s(0, x))=0$ by $2.10(1)$. The commutative law for $x+y$ follows from the fact that $x+y$ commutes with itself, since then $x+y=(0+x)+(y+0)=(0+y)+(x+0)=y+x$. Hence $-x$ is both a left and a right inverse for $x$. Now the associative law for $x+y$ is proved as follows:

$$
x+(y+z)=(0+x)+(z+y)=(0+z)+(x+y)=(x+y)+z .
$$


Therefore every member of $\mathscr{P}$ has abelian group terms and these terms commute with all other terms.

We define a ring associated to $\mathscr{P}$ as follows. Let $R$ be a representative set of unary terms of $\mathscr{P}$. The ring multiplication will be essentially composition of terms. Thus if $r(x), t(x) \in R$, then $(r t)(x)$ is defined to be the representative of $r(t(x))$. Addition is defined with the binary term $x+y$ from above: if $r(x), t(x) \in R$, then $(r+t)(x)$ is defined to be the representative of $r(x)+t(x)$. Of course, negation is defined by saying that $(-r)(x)$ equals the representative of $-(r(x))$. We define 0 by the representative of the term $0(x)=0$ for all $x$. The ring laws follow from the fact that $x+y,-x$ and 0 are abelian group terms of $\mathscr{P}$ which commute with all unary terms.

For each $\boldsymbol{A} \in \mathscr{P}$ we claim that $\boldsymbol{A}$ is term equivalent to the module $\langle A ;+,-, 0, r \in$ $R\rangle$. Clearly, this module is a reduct of $\boldsymbol{A}$, since all the defining operations are terms of $\boldsymbol{A}$. To see that all terms of $\boldsymbol{A}$ are expressible with module operations, choose an arbitrary $n$-ary term $f\left(x_{1}, \ldots, x_{n}\right)$. Since $f$ commutes with $x_{1}+\cdots+x_{n}$ on the array

$$
\left[\begin{array}{cccc}
x_{1} & 0 & \cdots & 0 \\
0 & x_{2} & \cdots & 0 \\
\vdots & & \ddots & \vdots \\
0 & 0 & \cdots & x_{n}
\end{array}\right],
$$

we get that $f\left(x_{1}, \ldots, x_{n}\right)=f_{1}\left(x_{1}\right)+\cdots+f_{n}\left(x_{n}\right)$ where $f_{i}\left(x_{i}\right)$ is the unary term in $R$ which represents $f\left(0, \ldots, 0, x_{i}, 0, \ldots, 0\right)$ with $x_{i}$ in the $i$-th position. This proves that $f$ equals a module operation. Hence, $A$ is term equivalent to a module.

We can finish the proof by showing that $\mathscr{P}$ is in fact a variety. (Any variety whose members are term equivalent to modules is itself term equivalent to a variety of modules as one can see by picking a generator for the variety.) Choose $\boldsymbol{A} \in \mathscr{P}$ arbitrarily and let $\theta$ be a congruence on $\boldsymbol{A}$. $\boldsymbol{A}$ is term equivalent to a module. Let $\boldsymbol{B}$ be the submodule of $A$ whose universe is $0 / \theta$ and let $\theta^{\prime}$ be the relative congruence of $A$ which has $B$ as a class (Lemma 2.6). Then $0 / \theta=B=0 / \theta^{\prime}$, so since $A$ is term equivalent to a module (and modules have regular congruences) we get $\theta=\theta^{\prime}$. This proves that every congruence on $\boldsymbol{A}$ is a relative congruence, so every homomorphic image of $\boldsymbol{A}$ belongs to $\mathscr{P}$. A prevariety closed under the formation of homomorphic images is a variety, so $\mathscr{P}$ is a variety.

In order to sum up, we turn our attention back to bialgebraic contexts and the case where $\underline{P}$ is finite. In the introduction, we gave a very natural construction of bialgebraic contexts for finite dimensional vector spaces and for finite Abelian groups. Our interest was to see for which classes of algebras we can obtain bialgebraic contexts in a similar way. At the beginning of this section, we explained that if such 
a construction yields a bialgebraic context for all finite members of the locally finite prevariety $\mathscr{P}$ then $\mathscr{P}$ must be normal and conormal. The result above says that then $\mathscr{P}$ is term equivalent to a variety of modules. Therefore, we can say that the 'nice' construction of bialgebraic contexts for finite dimensional vector spaces and finite Abelian groups is a very special case. With every reasonable generalization we can not go beyond varieties of modules.

THEOREM 2.12. Under the assumptions of Theorem 1.1, let $\mathscr{P}$ be generated by a finite algebra $\underline{P}$, and let $\mathbb{K}(\boldsymbol{A})$ be a bialgebraic context for every finite $\boldsymbol{A} \in \mathscr{P}$. Then $\mathscr{P}$ is term equivalent to a variety of modules and $\mathscr{Q}$ is a subcategory of a category which is term equivalent to a variety of modules. Furthermore, the duality between $\mathscr{P}$ and $\mathscr{Q}$ is full.

PROOF. We prove first that the duality must be full. Since the context $\mathbb{K}(A)$ is bialgebraic for all finite $\boldsymbol{A}$ in $\mathscr{P}$, the categories $\mathscr{P}_{\text {fin }}$ and $\mathscr{Q}_{\text {fin }}$ are normal. If $\boldsymbol{X}$ is a subalgebra of a finite algebra $Y \in \mathscr{Q}$, then there is a morphism $\varphi: Y \longrightarrow \underset{\sim}{P}$ such that $\varphi \neq 0_{Y P}$ and $\left.\varphi\right|_{X}=0_{X P}$ because $\mathscr{Q}_{\text {fin }}$ is normal. Now a direct application of Theorem $1.1 \widetilde{2}$ of [4] yields that the duality is full.

By Theorem 2.11 and Lemma 2.1 we know already that $\mathscr{P}$ is term equivalent to a variety of modules. Since the duality is full, $\mathscr{P}$ and $\mathscr{Q}$ are dually equivalent as categories; especially $\mathscr{Q}$ is normal and conormal. $\mathscr{Q}$ is not a prevariety since it does not contain the subalgebras which are not topologically closed. However, $\mathscr{Q}_{\text {fin }}=$ $\left(\operatorname{ISP}(\underset{\sim}{P})_{\text {fin }}\right.$, and with the same arguments as for $\mathscr{P}$ in Lemma 2.1 we can conclude that the prevariety ISP $(\underset{\sim}{P})$ is normal and conormal. Thus, it is term equivalent to a variety of modules, and $\mathscr{Q}$ is a subcategory of this variety.

\section{References}

[1] F. W. Anderson and K. R. Fuller, Rings and categories of modules (Springer, New York, 1974).

[2] D. M. Clark and B. A. Davey, 'When is a natural duality "good'?', Algebra Universalis, to appear.

[3] B. A. Davey, 'Duality theory on ten dollars a day', in: Algebras and orders (eds. I. G. Rosenberg and G. Sabidussi) (Kluwer Academic Publishers, Dordrecht, 1993) pp. 71-111.

[4] B. A. Davey and H. Werner, 'Dualities and equivalences for varieties of algebras', Colloquia Mathematica Societatis János Bolyai 33 (1983), 101-275.

[5] R. Freese and R. McKenzie, Commutator theory for congruence modular varieties, London Math. Soc. Lecture Notes 125 (Cambridge University Press, 1987).

[6] B. Ganter and R. Wille, 'Conceptual scaling', in: Applications of combinatorics and graph theory to the biological and social sciences (ed. F. Roberts) (Springer, New York, 1989) pp. 139-167.

[7] R. McKenzie, G. McNulty and W. Taylor, Algebras, lattices, varieties, volume 1 (Wadsworth and Brooks/Cole, Monterey, 1987).

[8] B. Mitchell, Theory of categories (Academic Press, 1965). 
[9] F. Vogt, Bialgebraic contexts (Ph. D. Thesis, TH Darmstadt, 1994; printed version: Shaker, Aachen, 1994).

[10] F. Vogt and R. Wille, 'Ideas of algebraic concept analysis', in: Information systems and data analysis (eds. H.-H. Bock, W. Lenski and M. M. Richter) (Springer, Heidelberg, 1994) pp. 193205.

[11] R. Wille, 'Restructuring lattice theory: an approach based on hierarchies of concepts', in: Ordered sets (ed. I. Rival) (Reidel, Dordrecht, 1982) pp. 445-470.

Department of Mathematical Sciences

University of Arkansas

Fayetteville, AR 72701

USA

e-mail: kearnes@comp.uark.edu
Fachbereich Mathematik Technische Hochschule Darmstadt Schloßgartenstraße 7 D-64289 Darmstadt Germany 